Article

\title{
Even Higher Order Fractional Initial Boundary Value Problem with Nonlocal Constraints of Purely Integral Type
}

\author{
Said Mesloub * and Faten Aldosari \\ Mathematics Department, College of Science, King Saud University, P.O. Box 2455, Riyadh 11451, Saudi Arabia; \\ Faten@ksu.edu.sa \\ * Correspondence: mesloub@ksu.edu.sa
}

Received: 31 January 2019; Accepted: 24 February 2019; Published: 1 March 2019

\begin{abstract}
In this paper, the a priori estimate method, the so-called energy inequalities method based on some functional analysis tools is developed for a Caputo time fractional $2 m$ th order diffusion wave equation with purely nonlocal conditions of integral type. Existence and uniqueness of the solution are proved. The proofs of the results are based on some a priori estimates and on some density arguments.
\end{abstract}

Keywords: energy inequality; integral conditions; fractional wave equation; existence and uniqueness; initial boundary value problem

\section{Introduction}

Classical initial boundary value problems for partial differential equations with integer and noninteger order have been widely studied during the last three decades by using different methods. One of the most important methods used and applied to linear and nonlinear partial differential equations with integer order supplemented with classical conditions is the functional analysis method. However, for equations with Caputo time fractional order and nonlocal conditions, there are only a few results obtained by using the mentioned method. The Caputo fractional derivative is a nonlocal operator since it is an integral which is a nonlocal operator. Caputo time fractional derivative can be used to model systems with memory, since it requires all the past history. Time fractional order partial differential equations play a great role in reducing the errors coming from the neglected parameters while modeling real life phenomena.

One of the most important classes of the above equations are the fractional diffusion-wave equations that have been studied and used in different branches of Science. Problem (1) constitutes a large class of time fractional diffusion wave equations of even order such as second and fourth order time fractional wave equations that have numerous applications in physics and engineering as mentioned below. In our problem, local conditions at 0 and 1 are replaced by other conditions on the moments of order $1,2, \ldots, 2 m-1$ which are non-local integral conditions. Although mathematical models in two and three-dimensions are of big significance for applications, most of the recent research articles are devoted to the fractional order diffusion wave equations in one-dimensional settings. These equations model, for example, propagation of mechanical waves in viscoelastic media [1-4], a non-Markovian diffusion process with memory [5], and a model governing the propagation of mechanical diffusive waves in viscoelastic media that exhibit a power-law creep [2-4].

For various applications of fractional calculus, the reader could refer to [4,6-13].

In the literature, many researchers used the functional analysis method to investigate the well posedness of initial boundary value problems for partial differential equations with time and space 
integer order having nonlocal conditions-we cite, for example, the references [14-17]. For the fractional diffusion wave equations case with higher order derivatives and classical boundary conditions, there are only few papers dealing with the existence and uniqueness of solution such as [18-20]. In this paper, an initial boundary value problem with purely nonlocal constraints of integral type for a Caputo time fractional $2 m$ th order diffusion wave equation is studied by applying the functional analysis method, the so-called energy inequality method based mainly on some a priori estimates and on the density of the range of the operator generated by the studied problem. This work can be considered as a contribution to the development of the functional analysis method used to prove the well posedness of problems with fractional order. The obtained results show the efficiency of this method to study the existence and uniqueness of solution for the time fractional order differential equations with nonlocal conditions.

This paper is organized as follows: in Section 2, we set our fractional initial boundary value problem. In Section 3, we give some preliminaries concerning the used function spaces, some useful tools and write down the given problem in its operator form. In Section 4, we establish an a priori estimate for the solution and deduce some consequences about the uniqueness of the solution and its dependence on the free term and the given data. Section 5 provides proofs of the main result concerning the solvability of the posed problem. We end our problem with conclusions.

\section{Problem Setting}

In the domain $Q=(0,1) \times(0, T)$ where $0 \leq T<\infty$, we consider the time fractional initial boundary problem of higher order with purely integral conditions

$$
\begin{cases}\mathcal{L} v=\partial_{t}^{\alpha+1} v+(-1)^{m} \theta(t) \frac{\partial^{2 m^{2}}}{\partial x^{2 m}}=f(x, t), & x \in(0,1) t \in(0, T), \\ l_{1} v=v(x, 0)=g(x), l_{2} v=v_{t}(x, 0)=h(x), & x \in(0,1), \\ \int_{0}^{1} x^{i} v(x, t) d x=0, i=\overline{0,2 m-1}, & t \in(0, T)\end{cases}
$$

where $\theta(t), f(x, t), g(x)$ and $h(x)$ are given functions that satisfy certain conditions which will be specified later on, and the operator $\partial_{t}^{\alpha+1}$ denotes the Caputo left fractional derivative of order $1+\alpha$ with $0<\alpha<1$ defined by (see [21])

$$
\partial_{t}^{\alpha+1} v(x, t)=\frac{1}{\Gamma(1-\alpha)} \int_{0}^{t} \frac{v_{\tau \tau}(x, \tau)}{(t-\tau)^{\alpha}} d \tau, t>0,
$$

where $\Gamma(1-\alpha)$ is the Gamma function.

The Riemann-Liouville integral of order $0<\alpha<1$ is defined by (see [21])

$$
D_{t}^{-\alpha} v(t)=\frac{1}{\Gamma(\alpha)} \int_{0}^{t} \frac{v(\tau)}{(t-\tau)^{1-\alpha}} d \tau
$$

Different properties of the Caputo fractional derivative and Riemann fractional-Liouville integral can be found in [21-23] and the references therein.

\section{Preliminaries}

In this section, we introduce some important lemmas and inequalities needed throughout the sequel, and write the posed problem in its operator form. 
Lemma 1 (Poincare type inequality). For $m \in \mathbb{N}$, we have

$$
\left\|\mathcal{I}_{x}^{2 m} v\right\|_{L^{2}(0, A)}^{2} \leq\left(\frac{A}{2}\right)^{2 m}\|v\|_{L^{2}(0, A)}^{2}
$$

where

$$
\mathcal{I}_{x}^{2 m} v=\int_{0}^{x} \int_{0}^{\xi_{1}} \ldots \int_{0}^{\xi_{2 m-1}} v(\eta, t) d \eta d \xi_{2 m-1} \ldots d \xi_{1}=\int_{0}^{x} \frac{(x-\xi)^{2 m-1}}{(2 m-1) !} v(\xi, t) d \xi .
$$

Lemma 2 ([24]). For any absolutely continuous function $J(s)$ on the interval [0,T], the following inequality hold

$$
J(s) \partial_{s}^{\alpha} J(s) \geq \frac{1}{2} \partial_{s}^{\alpha} J^{2}(s), \quad 0<\alpha<1 .
$$

Lemma 3 ([25]). Let $\varphi(t)$ be nonnegative and absolutely continuous on $[0, T]$, and, for almost all $t \in[0, T]$, satisfies the inequality

$$
\frac{d \varphi}{d t} \leq C(t) \varphi(t)+B(t),
$$

where the functions $C(t)$ and $B(t)$ are summable and nonnegative on $[0, T]$. Then,

$$
\begin{aligned}
\varphi(t) & \leq e^{\int^{t} C(\tau) d \tau}\left(\varphi(0)+\int_{0}^{t} B(\xi) \cdot e^{\int^{\tau} C(\tau) d \tau} d \xi\right) \\
& \leq e^{\int^{t} C(\tau) d \tau}\left(\varphi(0)+\int_{0}^{t} B(\tau) d \tau\right) .
\end{aligned}
$$

Lemma 3 can be generalized as

Lemma 4 ([24]). Let a nonnegative absolutely continuous function $\mathcal{Z}(t)$ satisfy the inequality

$$
\partial_{t}^{\alpha} \mathcal{Z}(t) \leq c_{1} \mathcal{Z}(t)+c_{2}(t), \quad 0<\alpha<1,
$$

for almost all $t \in[0, T]$, where $c_{1}$ is a positive constant and $c_{2}(t)$ is an integrable nonnegative function on $[0, T]$. Then,

$$
\mathcal{Z}(t) \leq \mathcal{Z}(0) E_{\alpha}\left(c_{1} t^{\alpha}\right)+\Gamma(\alpha) E_{\alpha, \alpha}\left(c_{1} t^{\alpha}\right) D_{t}^{-\alpha} c_{2}(t),
$$

where

$$
E_{\alpha}(x)=\sum_{n=0}^{\infty} \frac{x^{n}}{\Gamma(\alpha n+1)} \text { and } E_{\alpha, \alpha^{*}}(x)=\sum_{n=0}^{\infty} \frac{x^{n}}{\Gamma\left(\alpha n+\alpha^{*}\right)},
$$

are Mittag-Leffler functions.

Lemma 5 ([14]). Let $Z_{i}(\tau)(i=1,2,3)$ be nonnegative functions on the interval $[0, T], Z_{1}(\tau), Z_{2}(\tau)$ are integrable functions, and $\mathrm{Z}_{3}(\tau)$ is nondecreasing. Then,

$$
\int_{0}^{t} Z_{1}(\tau) d(\tau)+Z_{2}(t) \leq Z_{3}(t)+C \int_{0}^{t} Z_{2}(\tau) d(\tau)
$$

implies

$$
\int_{0}^{t} Z_{1}(\tau) d(\tau)+Z_{2}(t) \leq e^{C t} Z_{3}(t)
$$


Young's inequality with $\varepsilon$ : For any $\varepsilon>0$, we have the inequality

$$
\lambda \beta \leq \frac{1}{p}|\varepsilon \lambda|^{p}+\frac{p-1}{p}\left|\frac{\beta}{\varepsilon}\right|^{\frac{p}{p-1}}, \quad \lambda, \beta \in \mathbb{R}, p>1,
$$

where $\lambda$ and $\beta$ are nonnegative numbers.

A special case of (11) is the Cauchy inequality with $\varepsilon$ :

$$
\lambda \beta \leq \frac{\varepsilon}{2} \lambda^{2}+\frac{1}{2 \varepsilon} \beta^{2}, \quad \varepsilon>0,
$$

The solution of the problem (1) can be regarded as the solution of operator equation

$$
\mathcal{M} v=\mathcal{W}=(f, g, h),
$$

where $\mathcal{M}=\left(\mathcal{L}, l_{1}, l_{2}\right)$, and $\mathcal{M}: \mathcal{B} \longrightarrow \mathcal{Y}$ is an unbounded operator with domain of definition

$$
\mathcal{D}(\mathcal{M})=\left\{\begin{array}{l}
v \in L^{2}(Q), \partial_{t}^{\alpha+1} v, \frac{\partial^{2} m_{v}}{\partial x^{2 m}} \in L^{2}(Q), \\
\int_{0}^{1} x^{i} v d x=0, \quad i=\overline{0,2 m-1}, t \in(0, T),
\end{array}\right.
$$

such that $v$ satisfies the initial conditions and where $\mathcal{B}$ is a Banach space of functions $v$ endowed with the finite norm

$$
\|v\|_{\mathcal{B}}^{2}=\sup _{0 \leq t \leq T}\left(D_{t}^{\alpha-1}\left\|\mathcal{I}_{x}^{m} v_{t}(x, t)\right\|_{L^{2}(0,1)}^{2}+\int_{0}^{1} v^{2}(x, t) d x\right)
$$

and $\mathcal{Y}$ is Hilbert space constituting of the elements $\mathcal{W}=(f, g, h)$ equipped with the norm

$$
\|\mathcal{W}\|_{Y}^{2}=\|g\|_{L^{2}(0,1)}^{2}+\|h\|_{L^{2}(0,1)}^{2}+\|f\|_{L^{2}(Q)}^{2} .
$$

Here, $\mathcal{L}$ denotes the time fractional differential operator

$$
\mathcal{L}={ }^{C} \partial_{t}^{\alpha+1}+(-1)^{m} \theta(t) \frac{\partial^{2 m}}{\partial x^{2 m}} .
$$

\section{A Priori Estimate for the Solution and Uniqueness}

To prove the uniqueness of solution of problem (1), we establish an energy inequality for the solution from which we deduce the uniqueness of solution of the posed problem.

Theorem 1. Assume that the function $\theta(t)$ satisfies the conditions

$$
\text { i) } c_{2} \leq \theta(t) \leq c_{1}, \text { ii) } c_{4} \leq \theta^{\prime}(t) \leq c_{3}, \forall t \in[0, T] \text {, }
$$

where $c_{1}, c_{2}, c_{3}$, and $c_{4}$ are positive constants. Then, for any $v \in \mathcal{D}(\mathcal{M})$, there exists a positive constant $\mathcal{K}$ such that the following a priori estimate is satisfied:

$$
\begin{aligned}
& \sup _{0 \leq t \leq T}\left(D_{t}^{\alpha-1}\left\|\mathcal{I}_{x}^{m} v_{t}(x, t)\right\|_{L^{2}(0,1)}^{2}+\int_{0}^{1} v^{2}(x, t) d x\right) \\
\leq & \mathcal{K}\left(\|g\|_{L^{2}(0,1)}^{2}+\|h\|_{L^{2}(0,1)}^{2}+\|f\|_{L^{2}(Q)}^{2}\right),
\end{aligned}
$$

where $\mathcal{K}=\mathcal{K}(\eta, \delta, \rho)$ is given by

$$
\mathcal{K}=\rho\left(1+\frac{T^{\alpha}}{\Gamma(1+\alpha)}\right)
$$


with $\eta, \delta$ and $\rho$ are respectively given by (30), (34) and (37).

Proof. For $v \in D(L)$, we consider the scalar product in $L^{2}(0,1)$ of the differential equation in problem (1) and the integrodifferential operator $N v=2(-1)^{m} \mathcal{I}_{x}^{2 m} v_{t}$, we have

$$
\begin{aligned}
& 2(-1)^{m}\left(\partial_{t}^{\alpha+1} v, \mathcal{I}_{x}^{2 m} v_{t}\right)_{L^{2}(0,1)}+2\left(\theta(t) \frac{\partial^{2 m} v}{\partial x^{2 m}}, \mathcal{I}_{x}^{2 m} v_{t}\right)_{L^{2}(0,1)} \\
= & (\mathcal{L} v, N v)_{L^{2}(0,1)} .
\end{aligned}
$$

We separately consider the inner products on the left-hand side of Equation (20) and we integrate by parts and taking into account boundary and initial conditions in Problem (1), we obtain

$$
\begin{aligned}
2(-1)^{m}\left(\partial_{t}^{\alpha+1} v, \mathcal{I}_{x}^{2 m} v_{t}\right)_{L^{2}(0,1)} & =2(-1)^{m}\left(\partial_{t}^{\alpha} v_{t}, \mathcal{I}_{x}^{2 m} v_{t}\right)_{L^{2}(0,1)} \\
& =2\left(\partial_{t}^{\alpha}\left(\mathcal{I}_{x}^{m} v_{t}\right), \mathcal{I}_{x}^{m} v_{t}\right)_{L^{2}(0,1)} \\
2\left(\theta(t) \frac{\partial^{2 m} v}{\partial x^{2 m}}, \mathcal{I}_{x}^{2 m} v_{t}\right)_{L^{2}(0,1)} & =2 \int_{0}^{1} \theta(t) \frac{\partial^{2 m} v}{\partial x^{2 m}} \mathcal{I}_{x}^{2 m} v_{t} d x \\
& =2(-1)^{m}\left(\theta(t) \frac{\partial^{m} v}{\partial x^{m}}, \mathcal{I}_{x}^{m} v_{t}\right)_{L^{2}(0,1)} \\
& =2\left(\theta(t) v, v_{t}\right)_{L^{2}(0,1)} .
\end{aligned}
$$

Substitution of (21) and (22) into (20) yields

$$
\begin{aligned}
& 2\left(\partial_{t}^{\alpha}\left(\mathcal{I}_{x}^{m} v_{t}\right), \mathcal{I}_{x}^{m} v_{t}\right)_{L^{2}(0,1)}+2\left(\theta(t) v, v_{t}\right)_{L^{2}(0,1} \\
= & (\mathcal{L} v, N v)_{L^{2}(0,1)}=2(-1)^{m}\left(f, \mathcal{I}_{x}^{2 m} v_{t}\right)_{L^{2}(0,1)} .
\end{aligned}
$$

By Lemmas 1 and 2 and inequality (12), identity (23) reduces to

$$
\partial_{t}^{\alpha}\left\|\mathcal{I}_{x}^{m} v_{t}\right\|_{L^{2}(0,1)}^{2}+\left(\theta(t) v, v_{t}\right)_{L^{2}(0,1} \leq\|f\|_{L^{2}(0,1)}^{2}+\frac{1}{2^{m}}\left\|\mathcal{I}_{x}^{m} v_{t}\right\|_{L^{2}(0,1)}^{2} .
$$

Replacing $t$ by $\tau$, integrating with respect to $\tau$ from zero to $t$ and using given conditions, we obtain

$$
\begin{aligned}
& \int_{0}^{t} \partial_{\tau}^{\alpha}\left\|\mathcal{I}_{x}^{m} v_{\tau}\right\|_{L^{2}(0,1)}^{2} d \tau+\int_{0}^{t} \int_{0}^{1} \theta(\tau) v v_{\tau} d x d \tau \\
\leq & \int_{0}^{t}\|f(x, \tau)\|_{L^{2}(0,1)}^{2} d \tau+\frac{1}{2^{m}} \int_{0}^{t}\left\|\mathcal{I}_{x}^{m} v_{\tau}\right\|_{L^{2}(0,1)}^{2} d \tau .
\end{aligned}
$$

The second term on the left-hand side can be evaluated as

$$
\begin{aligned}
2 \int_{0}^{t} \int_{0}^{1} \theta(\tau) v v_{\tau} d x d \tau= & \int_{0}^{1} \theta(t) v^{2} d x-\theta(0) \int_{0}^{1} g^{2}(x) d x \\
& -\int_{0}^{t} \int_{0}^{1} \theta^{\prime 2} v^{2} d x d \tau .
\end{aligned}
$$


Hence, inequality (25) becomes

$$
\begin{aligned}
& \int_{0}^{t} \partial_{\tau}^{\alpha}\left\|\mathcal{I}_{x}^{m} v_{\tau}\right\|_{L^{2}(0,1)}^{2} d \tau+\frac{1}{2} \int_{0}^{1} \theta(t) v^{2} d x \\
\leq & \theta(0) \int_{0}^{1} g^{2}(x) d x+\int_{0}^{t} \int_{0}^{1} \theta^{\prime 2} v^{2} d x d \tau+\int_{0}^{t}\|f(x, \tau)\|_{L^{2}(0,1)}^{2} d \tau \\
& +\frac{1}{2^{m}} \int_{0}^{t}\left\|\mathcal{I}_{x}^{m} v_{\tau}\right\|_{L^{2}(0,1)}^{2} d \tau .
\end{aligned}
$$

Now, since

$$
\begin{aligned}
\int_{0}^{t} \partial_{\tau}^{\alpha}\left\|\mathcal{I}_{x}^{m} v_{\tau}\right\|_{L^{2}(0,1)}^{2} d \tau= & D^{\alpha-1}\left\|\mathcal{I}_{x}^{m} v_{t}\right\|_{L^{2}(0,1)}^{2} \\
& -\frac{t^{1-\alpha}}{(1-\alpha) \Gamma(1-\alpha)}\left\|\mathcal{I}_{x}^{m} h\right\|_{L^{2}(0,1)}^{2}
\end{aligned}
$$

evoking conditions (17) and using (28), we infer from (26) that

$$
\begin{aligned}
& D^{\alpha-1}\left\|\mathcal{I}_{x}^{m} v_{t}\right\|_{L^{2}(0,1)}^{2}+\|v\|_{L^{2}(0,1)}^{2} \\
\leq & \eta\left(\|h\|_{L^{2}(0,1)}^{2}+\|g\|_{L^{2}(0,1)}^{2}+\int_{0}^{t}\|f\|_{L^{2}(0,1)}^{2} d \tau\right. \\
& \left.+\int_{0}^{t}\left\|\mathcal{I}_{x}^{m} v_{\tau}\right\|_{L^{2}(0,1)}^{2} d \tau+\int_{0}^{t} \int_{0}^{1} v^{2}(x, \tau) d x d \tau\right),
\end{aligned}
$$

where

$$
\eta=\frac{\max \left(1, c_{1}, c_{3}^{2}, 2^{-m}, \frac{T^{1-\alpha} 2^{-m}}{(1-\alpha) \Gamma(1-\alpha)}\right)}{\min \left(1, c_{2}\right)}
$$

If, in Lemma 3, we set

$$
\varphi(t)=\int_{0}^{t} \int_{0}^{1} v^{2}(x, \tau) d x d \tau, \varphi^{\prime}(t)=\|v\|_{L^{2}(0,1)}^{2}, \text { and } \varphi(0)=0,
$$

then it yields

$$
\begin{aligned}
\int_{0}^{t} \int_{0}^{1} v^{2}(x, \tau) d x d \tau \leq & \operatorname{Te}^{\eta T} \eta\left(\int_{0}^{t}\|f(x, \tau)\|_{L^{2}(0,1)}^{2} d \tau+\int_{0}^{t}\left\|\mathcal{I}_{x}^{m} v_{\tau}\right\|_{L^{2}(0,1)}^{2} d \tau\right. \\
& \left.+\|g(x)\|_{L^{2}(0,1)}^{2}+\|h(x)\|_{L^{2}(0,1)}^{2}\right) .
\end{aligned}
$$


Consequently, (30) transforms to

$$
\begin{aligned}
& D^{\alpha-1}\left\|\mathcal{I}_{x}^{m} v_{t}\right\|_{L^{2}(0,1)}^{2}+\|v\|_{L^{2}(0,1)}^{2} \\
\leq & \delta\left(\int_{0}^{t}\|f(x, \tau)\|_{L^{2}(0,1)}^{2} d \tau+\int_{0}^{t}\left\|\mathcal{I}_{x}^{m} v_{\tau}\right\|_{L^{2}(0,1)}^{2} d \tau\right. \\
& \left.+\|g(x)\|_{L^{2}(0,1)}^{2}+\|h(x)\|_{L^{2}(0,1)}^{2}\right),
\end{aligned}
$$

where

$$
\delta=\max \left\{\eta, \eta^{2} T e^{\eta}\right\} .
$$

Now, by dropping the second term on the left-hand side of (33) then setting $\mathcal{Z}(t)=$ $\int_{0}^{t}\left\|\mathcal{I}_{x}^{m} v_{\tau}\right\|_{L^{2}(0,1)}^{2} d \tau, \partial_{t}^{\alpha} \mathcal{Z}(t)=D^{\alpha-1}\left\|\mathcal{I}_{x}^{m} v_{t}\right\|_{L^{2}(0,1)}^{2}$ in Lemma 4, we obtain

$$
\begin{aligned}
& \int_{0}^{t}\left\|\mathcal{I}_{x}^{m} v_{\tau}\right\|_{L^{2}(0,1)}^{2} d \tau \\
\leq & \delta \Gamma(\alpha) E_{\alpha, \alpha}\left(\delta t^{\alpha}\right)\left(D_{t}^{-\alpha-1}\|f\|_{L^{2}(0,1)}^{2}+\frac{T}{\alpha \Gamma(\alpha)}\|h\|_{L^{2}(0,1)}^{2}+\frac{T}{\alpha \Gamma(\alpha)}\|g\|_{L^{2}(0,1)}^{2}\right) \\
\leq & \delta \Gamma(\alpha) E_{\alpha, \alpha}\left(\delta t^{\alpha}\right) \max \left\{1, \frac{T}{\alpha \Gamma(\alpha)}\right\} \\
& \times\left(D_{t}^{-\alpha-1}\|f\|_{L^{2}(0,1)}^{2}+\|h\|_{L^{2}(0,1)}^{2}+\|g\|_{L^{2}(0,1)}^{2}\right) .
\end{aligned}
$$

Combination of (33) and (36) leads to

$$
\begin{aligned}
& D^{\alpha-1}\left\|\mathcal{I}_{x}^{m} v_{t}\right\|_{L^{2}(0,1)}^{2}+\|v\|_{L^{2}(0,1)}^{2} \\
\leq & \rho\left(\int_{0}^{t}\|f(x, \tau)\|_{L^{2}(0,1)}^{2} d \tau+D_{t}^{-\alpha-1}\|f\|_{L^{2}(0,1)}^{2}\right. \\
& \left.+\|h\|_{L^{2}(0,1)}^{2}+\|g\|_{L^{2}(0,1)}^{2}\right)
\end{aligned}
$$

where

$$
\rho=\delta \max \left(1, \delta \Gamma(\alpha) E_{\alpha, \alpha}\left(\delta t^{\alpha}\right) \max \left\{1, \frac{T}{\alpha \Gamma(\alpha)}\right\}\right) .
$$

It is obvious that

$$
\begin{aligned}
D_{t}^{-\alpha-1}\|f\|_{L^{2}(0,1)}^{2} & \leq \frac{t^{\alpha}}{\Gamma(1+\alpha)} \int_{0}^{t}\|f\|_{L^{2}(0,1)}^{2} d t \\
& \leq \frac{T^{\alpha}}{\Gamma(1+\alpha)} \int_{0}^{T}\|f\|_{L^{2}(0,1)}^{2} d t .
\end{aligned}
$$

Then, it follows from (37) and (38) that

$$
\begin{aligned}
& D^{\alpha-1}\left\|\mathcal{I}_{x}^{m} v_{t}\right\|_{L^{2}(0,1)}^{2}+\|v\|_{L^{2}(0,1)}^{2} \\
\leq & \mathcal{K}\left(\|f\|_{L^{2}(Q)}^{2}+\|h\|_{L^{2}(0,1)}^{2}+\|g\|_{L^{2}(0,1)}^{2}\right),
\end{aligned}
$$

where

$$
\mathcal{K}=\rho\left(1+\frac{T^{\alpha}}{\Gamma(1+\alpha)}\right)
$$


Observe that the right-hand side of (39) is independent of the variable $t$, so we are allowed to take the least upper bound of the left-hand side with respect to $t$ over $[0, T]$, and the a priori estimate (18) then follows and from which we deduce the uniqueness and continuous dependence of the solution on the input data of problem (1).

\section{Existence of Solution}

In this section, we prove the main result concerning the existence of solution of problem (1) The a priori estimate (18) shows that the unbounded operator $\mathcal{M}$ has an inverse $\mathcal{M}^{-1}: R(\mathcal{M}) \rightarrow \mathcal{B}$. Since $R(\mathcal{M})$ is a subset of $\mathcal{Y}$, we therefore can construct its closure $\overline{\mathcal{M}}$ in a manner that the estimate (18) holds for this extension and $R(\overline{\mathcal{M}})$ coincides with the whole space $\mathcal{B}$. Hence, the following:

Corollary 1. The operator $\mathcal{M}: \mathcal{B} \rightarrow \mathcal{Y}$ admits a closure (the proof is similar to that in [14]).

Estimate (18) can be then extended to

$$
\begin{aligned}
& \sup _{0 \leq t \leq T}\left(D_{t}^{\alpha-1}\left\|\mathcal{I}_{x}^{m} v_{t}(x, t)\right\|_{L^{2}(0,1)}^{2}+\int_{0}^{1} v^{2}(x, t) d x\right) \\
\leq & \mathcal{K}\left(\|g\|_{L^{2}(0,1)}^{2}+\|h\|_{L^{2}(0,1)}^{2}+\|f\|_{L^{2}(Q)}^{2}\right)
\end{aligned}
$$

for all $v \in D(\overline{\mathcal{M}})$.

It follows from (40) that the strong solution of problem (1) is unique, that is, $\overline{\mathcal{M}} v=\mathcal{Y}$. We also deduce from estimate (40) the following:

Corollary 2. $R(\overline{\mathcal{M}})$ is a closed subset in $\mathcal{Y}$ and $R(\mathcal{M})=R(\overline{\mathcal{M}})$ and $\overline{\mathcal{M}}^{-1}=\overline{\mathcal{M}^{-1}}$.

We are now ready to give the result of existence of the solution of problem (1).

Theorem 2. Suppose that conditions of Theorem 4.1 are satisfied. Then, for all $\mathcal{W}=(f, g, h) \in \mathcal{Y}$, there exists a unique strong solution $v=\overline{\mathcal{M}}^{-1} \mathcal{W}=\overline{\mathcal{M}}^{-1} \mathcal{W}$ of problem (1).

Proof. Estimate (40) asserts that, if a strong solution of (1) exists, it is unique and depends continuously on the data. Corollary 2 says that, in order to prove that problem (1) admits a strong solution for any $\mathcal{W}=(f, g, h) \in \mathcal{Y}$, it suffices to show that the closure of the range of the operator $\mathcal{M}$ is dense in $\mathcal{Y}$. To establish the existence of the strong solution of problem (1), we use a density argument. That is, we show that the range $R(\mathcal{M})$ of the operator $\mathcal{M}$ is dense in the space $\mathcal{Y}$ for every element $v$ in the Banach space $\mathcal{B}$. For this, we consider the following special case of density.

Theorem 3. Suppose that conditions of Theorem 1 are satisfied. If for all functions $v \in \mathcal{D}(\mathcal{M})$ such that $l_{1} v=v(x, 0)=0, l_{2} v=v_{t}(x, 0)=0$ and for some function $\psi \in L^{2}(Q)$, we have

$$
\int_{0}^{T}(\mathcal{L} v, \psi)_{L^{2}(0,1)} d t=0,
$$

then $\psi$ vanishes a.e in $Q$.

Proof. Identity (40) is equivalent to

$$
\int_{0}^{T}\left(\partial_{t}^{\alpha+1} v+(-1)^{m} \theta(t) \frac{\partial^{2 m} v}{\partial x^{2 m}}, \psi\right)_{L^{2}(0,1)} d t=0 .
$$


Assume that a function $\sigma(x, t)$ verifies conditions boundary and initial conditions in (1) and such that $\sigma, \sigma_{x}, \mathcal{I}_{t} \sigma, \mathcal{I}_{t} \mathcal{I}_{x}^{2 m} \sigma, \mathcal{I}_{t}^{2} \sigma$ and $\partial_{t}^{\beta+1} \sigma \in L^{2}(Q)$, we then set

$$
v(x, t)=\mathcal{I}_{t}^{2} \sigma=\int_{0}^{t} \int_{0}^{s} \sigma(x, z) d z d s .
$$

Equation (42) then becomes

$$
\int_{0}^{T}\left(\partial_{t}^{\alpha+1} \mathcal{I}_{t}^{2} \sigma+(-1)^{m} \theta(t) \cdot \frac{\partial^{2 m}}{\partial x^{2 m}}\left(\mathcal{I}_{t}^{2} \sigma\right), \psi\right)_{L^{2}(0,1)} d t=0 .
$$

We now introduce the function

$$
\psi(x, t)=\mathcal{I}_{t} \sigma+(-1)^{m} \mathcal{I}_{x}^{2 m} \mathcal{I}_{t} \sigma .
$$

Equation (44) then reduces to

$$
\begin{aligned}
& \int_{0}^{T}\left(\partial_{t}^{\alpha+1} \mathcal{I}_{t}^{2} \sigma, \mathcal{I}_{t} \sigma\right)_{L^{2}(0,1)} d t+\int_{0}^{T}\left(\partial_{t}^{\alpha+1} \mathcal{I}_{t}^{2} \sigma,(-1)^{m} \mathcal{I}_{x}^{2 m} \mathcal{I}_{t} \sigma\right)_{L^{2}(0,1)} d t \\
& +\int_{0}^{T}\left((-1)^{m} \theta(t) \cdot \frac{\partial^{2 m}}{\partial x^{2 m}}\left(\mathcal{I}_{t}^{2} \sigma\right), \mathcal{I}_{t} \sigma\right)_{L^{2}(0,1)} d t \\
& +\int_{0}^{T}\left(\theta(t) \cdot \frac{\partial^{2 m}}{\partial x^{2 m}}\left(\mathcal{I}_{t}^{2} \sigma\right), \mathcal{I}_{x}^{2 m} \mathcal{I}_{t} \sigma\right)_{L^{2}(0,1)} d t \\
& =0 .
\end{aligned}
$$

Recall that the function $\sigma$ satisfies boundary conditions in (1) and then, computing the inner products in (45), one has

$$
\begin{gathered}
\left(\partial_{t}^{\alpha+1} \mathcal{I}_{t}^{2} \sigma, \mathcal{I}_{t} \sigma\right)_{L^{2}(0,1)}=\left(\partial_{t}^{\alpha} \mathcal{I}_{t} \sigma, \mathcal{I}_{t} \sigma\right)_{L^{2}(0,1)}, \\
\geq \frac{1}{2} \partial_{t}^{\alpha}\left\|\mathcal{I}_{t} \sigma\right\|_{L^{2}(0,1)^{\prime}}^{2} \\
\left(\partial_{t}^{\alpha+1} \mathcal{I}_{t}^{2} \sigma,(-1)^{m} \mathcal{I}_{x}^{2 m} \mathcal{I}_{t} \sigma\right)_{L^{2}(0,1)}=\left(\partial_{t}^{\alpha} \mathcal{I}_{x}^{m} \mathcal{I}_{t} \sigma, \mathcal{I}_{x}^{m} \mathcal{I}_{t} \sigma\right)_{L^{2}(0,1)}, \\
\geq \frac{1}{2} \partial_{t}^{\alpha}\left\|\mathcal{I}_{x}^{m} \mathcal{I}_{t} \sigma\right\|_{L^{2}(0,1)^{\prime}}^{2} \\
\left((-1)^{m} \theta(t) \cdot \frac{\partial^{2 m}}{\partial x^{2 m}}\left(\mathcal{I}_{t}^{2} \sigma\right), \mathcal{I}_{t} \sigma\right)_{L^{2}(0,1)} \\
=\left(\theta(t) \frac{\partial^{m}}{\partial x^{m}}\left(\mathcal{I}_{t}^{2} \sigma\right), \frac{\partial^{m}}{\partial x^{m}}\left(\mathcal{I}_{t} P\right)\right)_{L^{2}(0,1)} \\
=\frac{1}{2} \frac{d}{d t} \int_{0}^{1} \theta(t)\left(\frac{\partial^{m}}{\partial x^{m}}\left(\mathcal{I}_{t}^{2} \sigma\right)\right)^{2} d x-\frac{1}{2} \int_{0}^{1} \theta^{\prime}(t)\left(\frac{\partial^{m}}{\partial x^{m}}\left(\mathcal{I}_{t}^{2} \sigma\right)\right)^{2} d x,
\end{gathered}
$$




$$
\begin{aligned}
& \left(\theta(t) \cdot \frac{\partial^{2 m}}{\partial x^{2 m}}\left(\mathcal{I}_{t}^{2} \sigma\right), \mathcal{I}_{x}^{2 m} \mathcal{I}_{t} \sigma\right)_{L^{2}(0,1)} \\
= & \left(\theta(t)\left(\mathcal{I}_{t}^{2} \sigma\right), \mathcal{I}_{t} \sigma\right)_{L^{2}(0,1)}=\frac{1}{2} \frac{d}{d t} \int_{0}^{1} \theta(t)\left(\mathcal{I}_{t}^{2} \sigma\right)^{2} d x \\
& -\frac{1}{2} \int_{0}^{1} \theta^{\prime}(t)\left(\mathcal{I}_{t}^{2} \sigma\right)^{2} d x .
\end{aligned}
$$

Evoking (47)-(51), replace $t$ by $\tau$, integrating with respect to $\tau$ from zero to $t$ and using conditions (17), we obtain

$$
\begin{aligned}
& D_{t}^{\alpha-1}\left\|\mathcal{I}_{t} \sigma\right\|_{L^{2}(0,1)}^{2}+D_{t}^{\alpha-1}\left\|\mathcal{I}_{x}^{m} \mathcal{I}_{t} \sigma\right\|_{L^{2}(0,1)}^{2}+\int_{0}^{1}\left(\frac{\partial^{m}}{\partial x^{m}}\left(\mathcal{I}_{t}^{2} \sigma\right)\right)^{2} d x \\
& +\int_{0}^{1}\left(\mathcal{I}_{t}^{2} \sigma\right)^{2} d x \\
\leq & \frac{c_{3}}{\min \left(1, c_{1}\right)}\left(\int_{0}^{t} \int_{0}^{1}\left(\frac{\partial^{m}}{\partial x^{m}}\left(\mathcal{I}_{\tau}^{2} \sigma\right)\right)^{2} d x d \tau+\int_{0}^{t} \int_{0}^{1}\left(\mathcal{I}_{\tau}^{2} \sigma\right)^{2} d x d \tau\right) .
\end{aligned}
$$

By dropping the first two terms on the left-hand side of (50) and applying Gronwall's Lemma 5, by setting $Z_{1}(t)=0, Z_{3}(t)=0$ and

$$
Z_{2}(t)=\int_{0}^{1}\left(\frac{\partial^{m}}{\partial x^{m}}\left(\mathcal{I}_{t}^{2} \sigma\right)\right)^{2} d x+\int_{0}^{1}\left(\mathcal{I}_{t}^{2} \sigma\right)^{2} d x
$$

we have

$$
\int_{0}^{1}\left(\frac{\partial^{m}}{\partial x^{m}}\left(\mathcal{I}_{t}^{2} \sigma\right)\right)^{2} d x+\int_{0}^{1}\left(\mathcal{I}_{t}^{2} \sigma\right)^{2} d x \leq 0
$$

for all $t \in[0, T]$. Hence, $\psi=0$ a.e in $Q$.

To complete the proof of Theorem 2, and prove the density $(\overline{R(\mathcal{M})}=\mathcal{Y})$ in a general case, suppose that, for some element $\left(F_{1}, \theta_{1}, \theta_{2}\right) \in R(\mathcal{M})^{\perp}$, we have

$$
\int_{0}^{T}\left(\mathcal{L} v, F_{1}\right)_{L^{2}(0,1)} d s+\left(l_{1} v, \theta_{1}\right)_{L^{2}(0,1)}+\left(l_{2} v, \theta_{2}\right)_{L^{2}(0,1)}=0,
$$

and then we prove that $F_{1}=0, \theta_{1}=0, \theta_{2}=0$. If we put $v \in D(\mathcal{M})$ satisfying conditions $l_{1} v=v(x, 0)=0$ and $l_{2} v=v_{t}(x, 0)=0$ into (53), we obtain

$$
\int_{0}^{T}\left(\mathcal{L} v, F_{1}\right)_{L^{2}(0,1)} d s=0, \forall v \in D(\mathcal{M}) .
$$

By Theorem 3, Equation (54) implies that $F_{1}=0$ a.e in $Q$. Then, (53) becomes

$$
\left(l_{1} v, \theta_{1}\right)_{L^{2}(0,1)}+\left(l_{2} v, \theta_{2}\right)_{L^{2}(0,1)}=0 \forall \theta \in D(\mathcal{M}) .
$$

Since the range of the trace operator $l_{1}$ and $l_{2}$ is dense in $L^{2}(0,1)$, it follows then from (55) that $\theta_{1}=0, \theta_{2}=0$. This ends the proof of Theorem 2 . 


\section{Conclusions}

The existence and uniqueness of a generalized solution for a higher order fractional diffusion wave equation in Caputo sense subject to initial and weighted integral boundary conditions are established. It is found that the method of energy inequalities is successfully applied to obtaining a priori bounds for the solution of fractional initial boundary value problems of higher order with nonlocal constraints as in the classical case. The obtained results will contribute to the development of the functional analysis method and enrich the existing non-extensive literature on the non local fractional mixed problems in the Caputo sense.

Author Contributions: Both authors have contributed equally to this paper.

Funding: The authors would like to extend their sincere appreciation to the Deanship of Scientific Research at King Saud University for its funding this Research group No. (RG-117).

Conflicts of Interest: The authors declare no conflict of interest.

\section{References}

1. Daftardar-Gejji, V.; Jafari, H. Boundary value problems for fractional diffusion-wave equations. Aust. J. Math. Anal. Appl. 2006, 3, 1-18.

2. Mainardi, F. Fractional relaxation-oscillation and fractional diffusion wave phenomena. Chaos Solitons Fractals 1996, 7, 1461-1477. [CrossRef]

3. Mainardi, F.; Luchko, Y.; Pagnini, G. The fundamental solution of the space-time fractional diffusion equation. Fract. Calc. Appl. Anal. 2001, 4, 153-192.

4. Mainardi, F.; Paradisi, P. Fractional diffusive waves. J. Comput. Acoust. 2001, 9, 1417-1436. [CrossRef]

5. Metzler, R.; Klafter, J. Boundary value problems for fractional diffusion equations. Physica A 2000, 298, 107-125. [CrossRef]

6. Carlson, G.E.; Halijak, C.A. Simulation of the fractional derivative operator vG and the fractional integral operator 1/v/S. Kansas State Univ. Bull. 1961, 45, 21-22.

7. Engheta, N. On fractional calculus and fractional multipoles in electromagnetism. IEEE Trans. Antennas Propag. 1996, 44, 554-566. [CrossRef]

8. Gorenflo, R. Abel integral equations with special emphasis on applications. In Lectures in Mathematical Sciences; University of Tokyo: Tokyo, Japan, 1996; Volume 13.

9. Hilfer, R. Applications of Fractional Calculus in Physics; World Scientific: Singapore, 2000.

10. Kaplan, T.; Gray, L.J.; Liu, S.H. Self-affine fractal model for a metal-electrolyte interface. Phys. Rev. B 1987, 35, 5379-5381. [CrossRef]

11. Nigmatullin, R. Realization of the generalized transfer equation in a medium with fractional geometry. Phys. Status B Basic Res. 1986, 133, 425-430. [CrossRef]

12. Marin, M.; Ochsner, A. The effect of a dipolar structure on the Holder stability in Green Naghdi thermoelasticity. Contin. Mech. Thermodyn. 2017, 29, 1365-1374. [CrossRef]

13. Anastassiou, G.; Argyros, I. Intelligent Numerical Methods: Applications to Fractional Calculus, Studies in Computational Intelligence; Springer: Heidelberg, Germany, 2016.

14. Mesloub, S. A nonlinear nonlocal mixed problem for a second order parabolic equation. J. Math. Anal. Appl 2006, 316, 189-209. [CrossRef]

15. Mesloub, S. On a singular two dimensional nonlinear evolution equation with non local conditions. Nonlinear Anal. 2008, 68, 2594-2607. [CrossRef]

16. Mesloub, S.; Bouziani, A. On a class of singular hyperbolic equations with a weighted integral condition. Int. J. Math. Math. Sci. 1999, 22, 511-519. [CrossRef]

17. Yurchuk, N.I. Mixed problem with an integral condition for certain parabolic equations. Differential'nye Uravneniya 1986, 22, 2117-2126.

18. Amanov, D. Solvability of boundary value problems for higher order differential equation with fractional derivative. Probl. Comput. Appl. Math. Tashkent Uzbikistan 2009, 121, 55-62.

19. Amanov, D.; Ashyralev, A. Initial-Boundary Value Problem for Fractional Partial Differential Equations of Higher Order. Abstr. Appl. Anal. 2012, 2012, 973102. [CrossRef] 
20. Mesloub, S.; Obaid, A. On a singular nonlocal time fractional order mixed problem with a memory term. Math. Methods Appl. Sci. 2018, 41, 4676-4690. [CrossRef]

21. Podlubny, I. Fractional Differential Equations; Academic Press: San Diego, CA, USA, 1999.

22. Kilbas, A.A.; Srivastava, H.M.; Trujillo, J.J. Theory and Applications of Fractional Differential Equations; Elsevier: Amsterdam, The Netherlands, 2006.

23. Samko, S.G.; Kilbas, A.A.; Marichev, O.I. Fractional Integrals and Derivatives, Theory and Applications. Minsk Nauka I Tekhnika 1993, 3, 397-414.

24. Alikhanov, A.A. A Priori Estimates for Solutions of Boundary Value Problems for Fractional-Order Equations. arXiv 2010, arXiv:1105.4592v1.

25. Ladyzhenskaya, O.A. The Boundary Value Problems of Mathematical Physics; Springer: New York, NY, USA, 1985.

(C) 2019 by the authors. Licensee MDPI, Basel, Switzerland. This article is an open access article distributed under the terms and conditions of the Creative Commons Attribution (CC BY) license (http:/ / creativecommons.org/licenses/by/4.0/). 